$$
\begin{aligned}
& \text { التحليل الديناميكي للجسور الصندوقية بسيطة الاسناد والمحتوية على حواجز وسطية }
\end{aligned}
$$

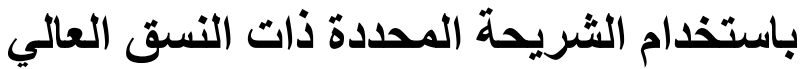

$$
\begin{aligned}
& \text { ريفان ناهض وديع ماجنير } \\
& \text { أياد امجد عبد الرزاق } \\
& \text { أستاذ مساعد }
\end{aligned}
$$

\title{
الخلاصة
}

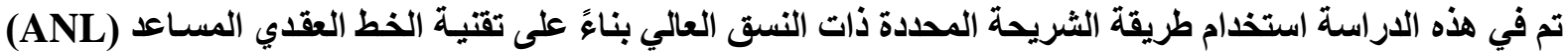

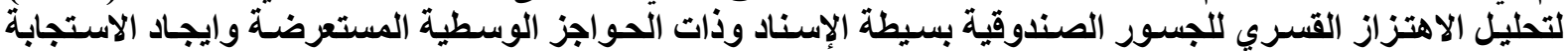

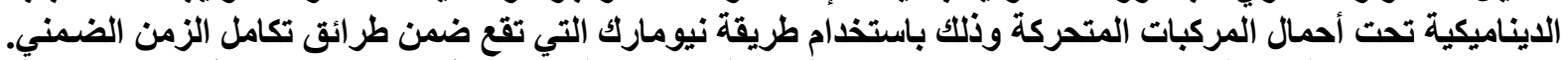

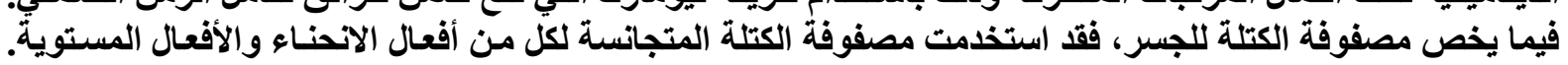

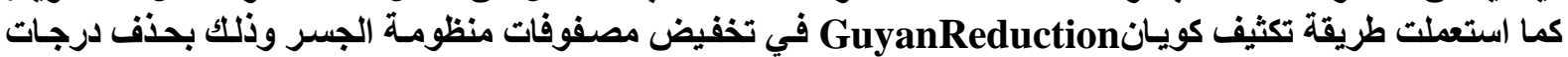

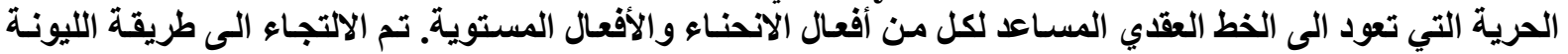
Flexibility Method

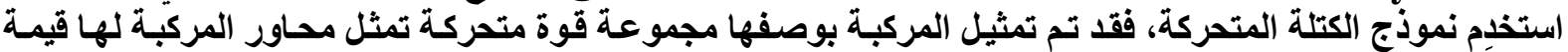
تساوي وزن محور المركبة.

الكلمات الدالة: جسر صندوقي، استجابة ديناميكية، شريحة محددة، حولجز وسطية، اهتزاز قسري، قوة متحركة.

\section{Dynamic Analysis of Simply Supported Box Girder Bridges with Intermediate Diaphragm Using Higher Order Finite Strip}

\author{
A.A. Abdul-Razzak \\ Assistant Professor
}

\author{
A.N. Revan \\ M. Sc.
}

\begin{abstract}
In The present work the method of Higher Order Finite Strip, based on Auxiliary Nodular Line (ANL) technique, was utilized to analyze the forced vibration of the Simply Supported Box Girder Bridges with cross intermediate diaphragms, Also to find out the dynamic response under moving vehicles loads by means of Newmark's method which falls within the Implicit Time Integration methods. The Bridge Mass Matrix and the Consistent Mass Matrix was used for both bending and in plane actions. While the Guyan Reduction method was used for reducing the Bridge System Matrix through omitting the Freedom Degrees belonging to the Auxiliary Nodular Line for both bending and in plane actions, In order to enable the Higher Order Finite Strip method for analyzing the box girder with cross intermediate diaphragms. vehicle model were used in the analysis is the Moving Force (MF) where the vehicle represents a no. of moving force with a value equal to the weight of the vehicle wheel.
\end{abstract}




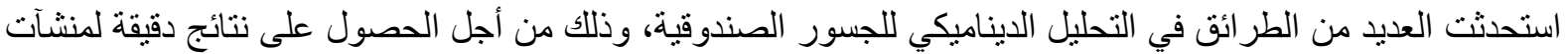

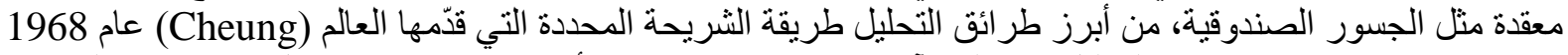
فهي تعد طريقة كفوة وبة وبسيطة لتحليل هذه المنشآت فهي طريقة هجينة إذ أنها تحتفظ بمميز ات كل من طريقة الصفائح

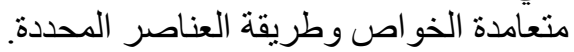

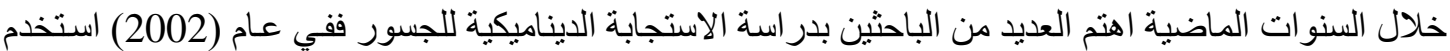
تائر Yuan-sheng et al.

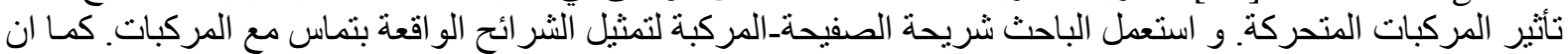

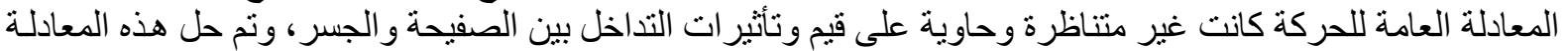
بالتكامل العددي للزمن باستعمال طريقة نيو مارك Newmark ومن مقارنة النتائج مع طريقة Method (SIM)

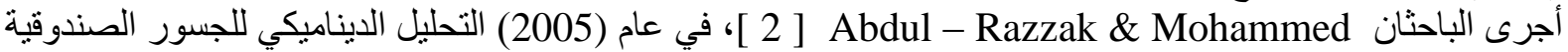

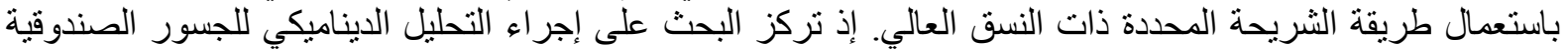

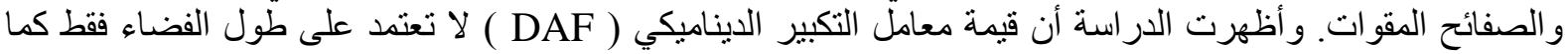

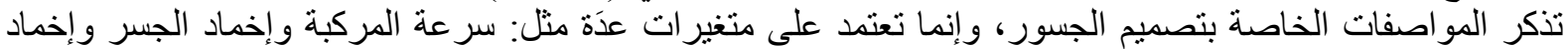

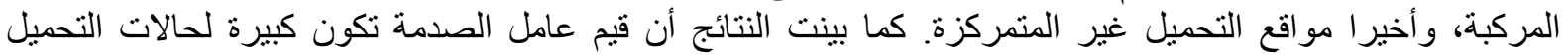

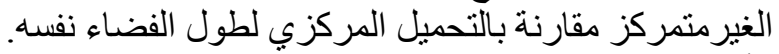

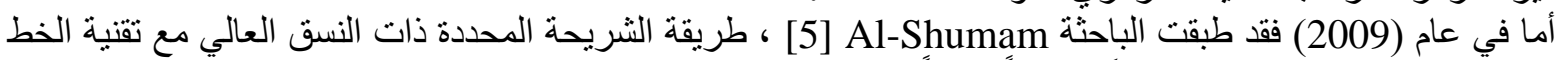

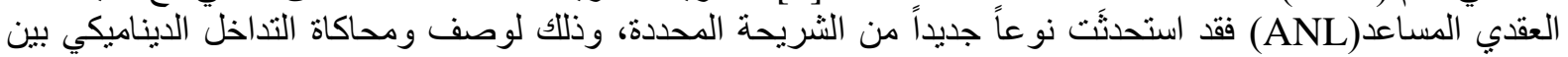

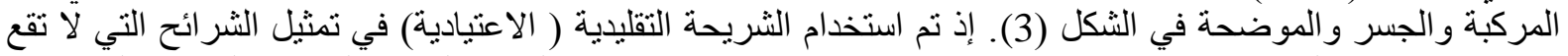

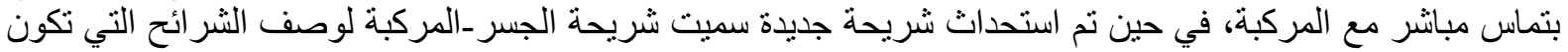

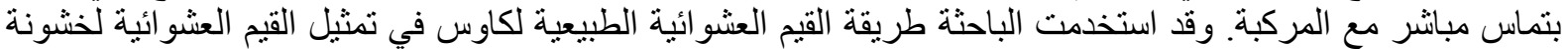

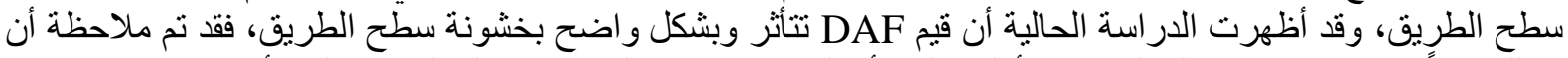

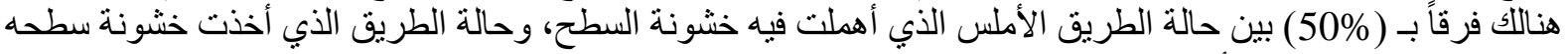

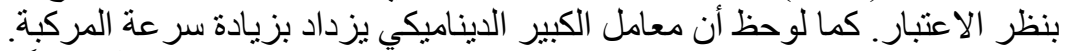

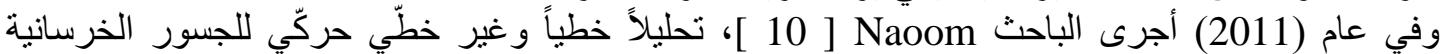

المسلحة باستخدام طريقة العناصر المحددة ذات العناصر ثلاثية الابعاد وعشرين عقدة (

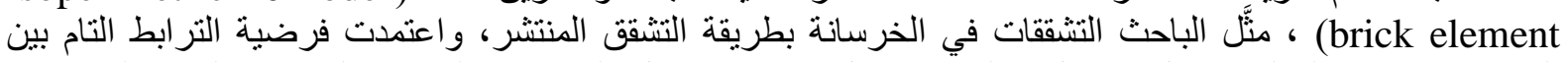

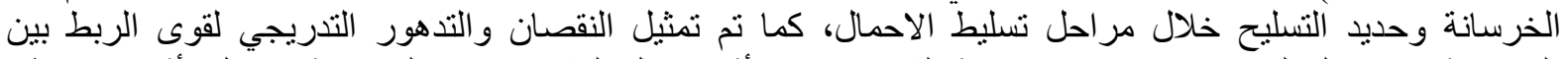

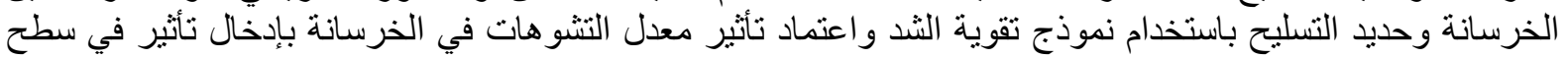

الخضوع في نموذج الضغط.

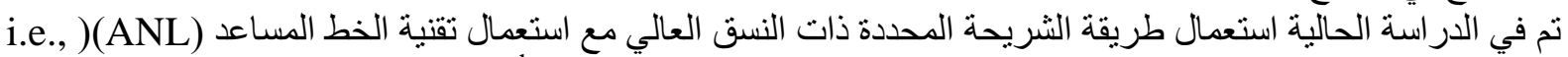

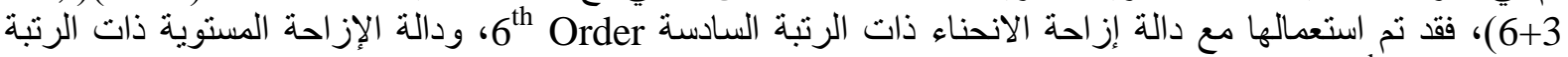

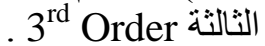

\section{2. صياغة الشريحة المحددة ذات النسق العالي: 1.2 أشتقاق مصفوفة الصلابة:}

يُعَبَّر عن دالة الاز احة للشريحة تحت أفعال الانحناء بالصيغة الآتية:[1,2,4]

$w(x, y)=\sum_{m=1}^{r}\left[C^{b}\right]\left\{W_{m}^{b}\right\} \sin k_{m} y$

$$
\begin{aligned}
& \text { y } \\
& \text { r r }
\end{aligned}
$$$$
k_{m}=m \pi / a
$$$$
\text { a }
$$ 
[C] مصفوفة المعاملات (من الشروط الحدودية) وهي دالة لـ x فقط، ويعبر عنها بما يلي [2]:

$C_{1}=1-39 S^{2}+162 S^{3}-276 S^{4}+216 S^{5}-64 S^{6}$

$C_{2}=x\left(1-8 S+25 S^{2}-38 S^{3}+28 S^{4}-8 S^{5}\right)$

$C_{3}=48 S^{2}-224 S^{3}+432 S^{4}-384 S^{5}+128 S^{6}$

$C_{4}=x\left(-8 S+32 S^{2}-40 S^{3}+16 S^{4}\right)$

$C_{5}=x^{2}\left(2-12 S+26 S^{2}-24 S^{3}+8 S^{4}\right)$

$C_{6}=-9 S^{2}+62 S^{3}-156 S^{4}+168 S^{5}-64 S^{6}$

$C_{7}=x\left(S-7 S^{2}+18 S^{3}-20 S^{4}+8 S^{5}\right)$

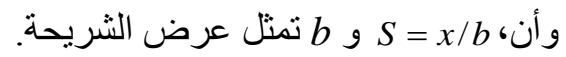

بالنسبة للثريحة متعامدة الخو اص، فإن متجه العزم و التقوس يمكن ان تعطى بالصيغة التالية:

$\{\phi\}=\sum_{m=1}^{r}\left[B_{m}^{b}\right]\left\{W_{m}^{b}\right\}$

$\{M\}=\left[D^{b}\right]\{\phi\}$

[مثل مصفوفة المرونة لأفعال الانحناء $\left[D^{b}\right]$.

أن الطاقة الكامنة الكلية للثريحة نتيجة الحمل الخارجي (x , y

$U^{b}=\frac{1}{2} \int_{0}^{a} \int_{0}^{b}\{M\}^{T}\{\phi\} d x d y-\int_{0}^{a} \int_{0}^{b} q_{e}(x, y)\{W\}^{b} d x d y$

بتعويض المعادلتين (3) و (4) في المعادلة (5)، فإن المعادلة الناتجة ولعدد تو افقي معين m توضح بالصيغة التالية: $\left[S_{m}^{b}\right]\left\{W_{m}^{b}\right\}=\left\{F_{m}^{b}\right\}$

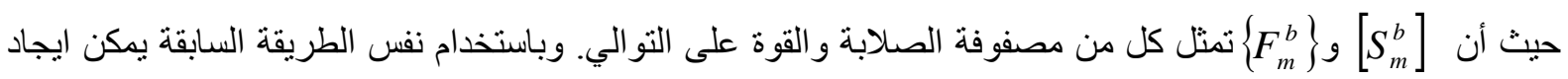

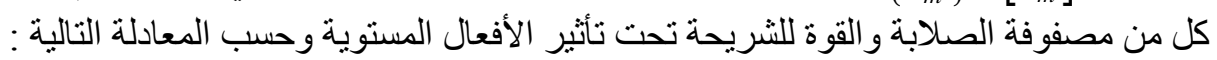

$\left[S_{m}^{p}\right]\left\{W_{m}^{p}\right\}=\left\{F_{m}^{p}\right\}$

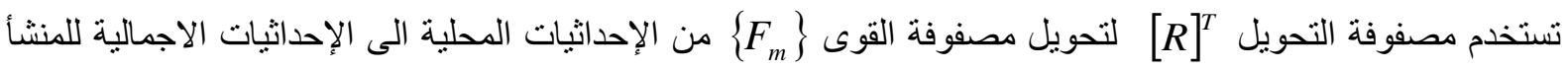
وكذلك ربط كل من افعال الانحناء والافعال المستوية وبالصيغة النتالية: $\left\{\bar{F}_{m}\right\}$

$\left\{F_{m}\right\}=[R]^{T}\left\{\bar{F}_{m}\right\}$

$\left\{W_{m}\right\}=[R]^{T}\left\{\bar{W}_{m}\right\}$

$\left[S_{m}\right]\left\{W_{m}\right\}=\left\{F_{m}\right\}$

$\left[\bar{S}_{m}\right]\left\{\bar{W}_{m}\right\}=\left\{\bar{F}_{m}\right\}$

بتعويض المعادلتين (8) و (9) في المعادلة (10) ينتج مايلي :

$$
\left[\bar{S}_{m}\right]=[R]\left[S_{m}\right][R]^{T}
$$


[ تمثل مصفوفة الصلابة بالإحداثيات الإجمالية للمنشأ.

2.2 أشتقاق مصفوفة الكتنة المتجاتسة:

يمكن التعبير عن دالة الإزاحة لأي شريحة كما يأتي] 6 ] :

$\{f\}=[B]\{W\}=\sum_{m=1}^{r}\left[B_{m}\right]\left\{W_{m}\right\}$

$\left[B_{m}\right]=[C] Y_{m}=[C] \sin k_{m} y$

إن [C] تمثل مصفوفة معاملات دالة الإز احة لأفعال الإنحناء أو الافعال المستوية ـ وبالتالي فإن مصفوفة الكتلة المتجانسة $[M]_{m n}=\int \rho h[B]_{m}^{T}[B]_{n} d($ area $)$

لكل وحدة تعرف بالمعادلة التالية [6] :

حيث أن م تمثل الكتلة لكل وحدة حجم و أن h تمثل سمك الثريحة.

بالإعتماد على المعادلة (15)، فإن مصفوفة الكتلة المتجانسة للثريحة ذات النسق العالي تحت تأثير الإنحناء تعطى $\left[M^{b}\right]_{m}=\int_{0}^{b}[C]^{T}[C] \int_{0}^{a} \rho h \sin ^{2} k_{m} y d x d y$

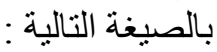

ويمكن أثتقاق مصفوفة الكتلة المتجانسة للشريحة تحت تأثير الافعال المستوية بنفس الطريقة السابقة ويمكن التعبير عن مصفوفة $[B]_{m}$ للافعال المستوية بالثكل التالي:

$[B]_{m}=\left[\begin{array}{cccccccc}C_{1} Y_{m} & 0 & C_{2} Y_{m} & 0 & C_{3} Y_{m} & 0 & C_{4} Y_{m} & 0 \\ 0 & \frac{a}{m \pi} C_{1} Y_{m}^{\prime} & 0 & \frac{a}{m \pi} C_{2} Y_{m}^{\prime} & 0 & \frac{a}{m \pi} C_{3} Y_{m}^{\prime} & 0 & \frac{a}{m \pi} C_{4} Y_{m}^{\prime}\end{array}\right]$

إن قيمة كل من $C_{1}, C_{2}, C_{3}, C_{4}$ : تعرف بالمعادلات التالية

$C_{1}=1-5 S+8 S^{2}-4 S^{3}$

$C_{2}=4 S-4 S^{2}$

$C_{3}=\left(-2 S+6 S^{2}-4 S^{3}\right) b$

$C_{4}=S-4 S^{2}+4 S^{3}$

$$
\text { إذ أن (S) تمثل : }
$$

\section{3. معاملة الحواجز المستعرضة:}

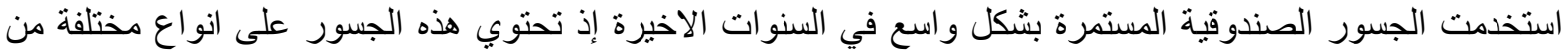

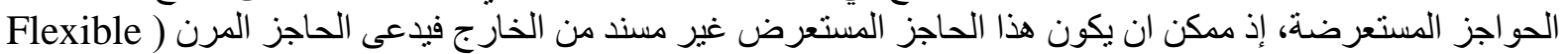
.(Diaphragm في البدء يفرض ان الحاجز المستعرض يرتبط بالمقطع الصندوقي بنقاط مختارة مسبقاً تسمى بالنقاط المقيدة ( Restrained . [9,3] (Points ويمكن التعبير عن القوى المقيدة سو اء كانت تابعة لحاجز مستعرض او لمسند خارجي بالآتي [9,3] . 


$$
\{r\}_{i}=\left[H_{1}, \mathrm{~V}_{1}, \mathrm{~T}_{1}, H_{2}, \mathrm{~V}_{2}, \mathrm{~T}_{2}, \ldots \ldots, H_{N}, \mathrm{~V}_{\mathrm{N}}, \mathrm{T}_{\mathrm{N}}\right]^{\mathrm{T}}
$$

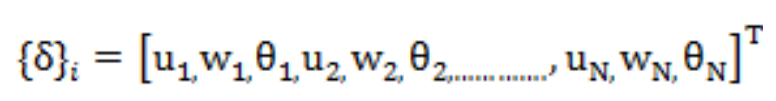$$
\text { وتبعا لهذه القوى فان الاز احات ستكون على النحو الآتي: }
$$

$[f]\{r\}=\{\delta\}$

وبتطبيق الثروط التو افقية للنشوه Displacement Compatibility Conditions

و المصفو فة الآتية تمثل مصفوفة الليونة للمقطع المقيد بحاجز مستعرض او المقطع المثبت خارجيا:

$$
[f]=\left[\begin{array}{ccc}
{\left[\mathrm{f}_{11}\right]} & {\left[\mathrm{f}_{12}\right] \ldots \ldots} & {\left[\mathrm{f}_{1 \mathrm{~L}}\right]} \\
{\left[\mathrm{f}_{21}\right]} & {\left[\mathrm{f}_{22}\right] \ldots . .} & {\left[\mathrm{f}_{2 \mathrm{~L}}\right]} \\
\vdots & \vdots & \\
{\left[\mathrm{f}_{\mathrm{L} 1}\right]} & {\left[\mathrm{f}_{\mathrm{L} 2}\right] \ldots . .} & {\left[\mathrm{f}_{\mathrm{LL}}\right]}
\end{array}\right]
$$

إذ أن $1 f_{\text {LLL }}$ تمثل مصفوفة الليونة (Flexibility Matrix) للمقطع المقيد بحاجز مستعرض .

\section{3 طريقة الليونة لهيكل التقوية الوسطي القابل للتشوه :}

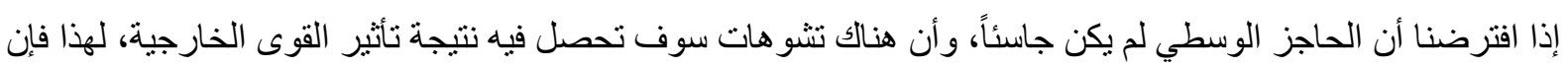

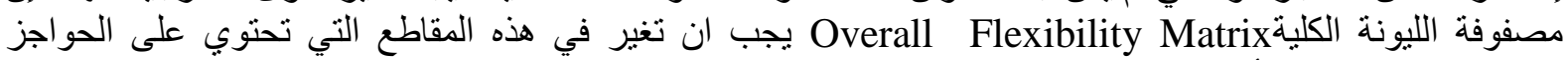

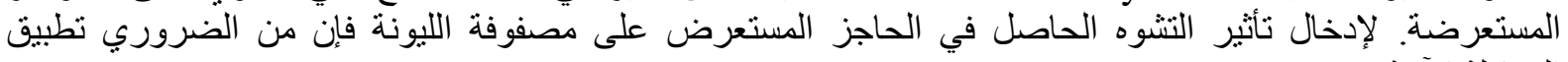
$[F]=\left[\begin{array}{ccccc}{\left[f_{11}\right]} & {\left[f_{12}\right]} & \cdot & \cdot & {\left[f_{11}\right]} \\ {\left[f_{21}\right]} & {\left[f_{22}\right]} & \cdot & \cdot & {\left[f_{21}\right]} \\ \cdot & \cdot & \cdot & \cdot & \cdot \\ \cdot & \cdot & \cdot & \cdot & \cdot \\ \cdot & \cdot & \cdot & \cdot & {\left[f_{11}\right]}\end{array}\right]+\left[\begin{array}{ccccc}{\left[f_{11}^{\circ}\right]} & 0 & 0 & 0 & 0 \\ 0 & {\left[f_{22}^{\circ}\right]} & 0 & 0 & 0 \\ 0 & 0 & \cdot & 0 & 0 \\ 0 & 0 & 0 & \cdot & 0 \\ 0 & 0 & 0 & 0 & {\left[f_{11}^{\prime}\right]}\end{array}\right]$ المعادلة الآتية] [9,3] . لالئل il [ ]

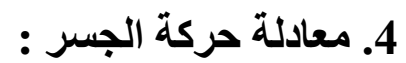

إنَّ معادلة الاهتزاز القسري التي ثُعُرف حركة الجسر (BridgeMotion) في الاتجاه الطولي يمكن التعبير عنها بمـا يأتي $[M]_{m}\{\ddot{\delta}\}_{m}+[C]_{m}\{\dot{\delta}\}_{m}+[K]_{m}\{\delta\}_{m}=\{F\}_{m}$

حيث أن:

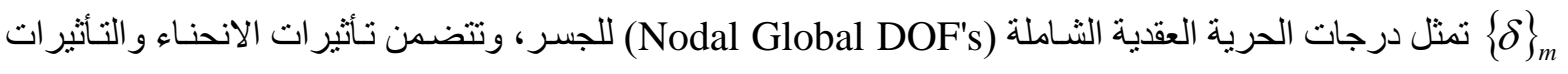

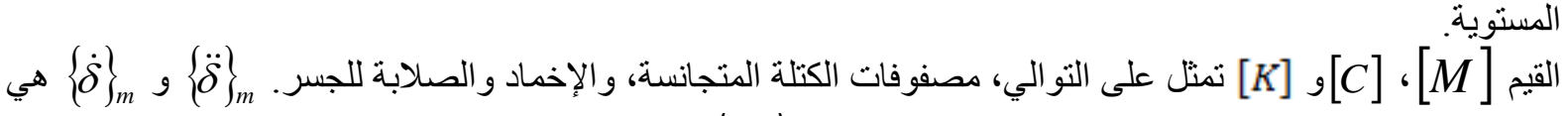

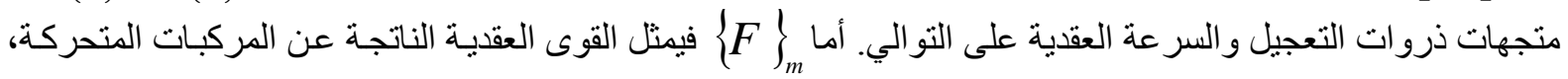
وقيمتها تتغير حسب حركة المركبة على الجسر. 


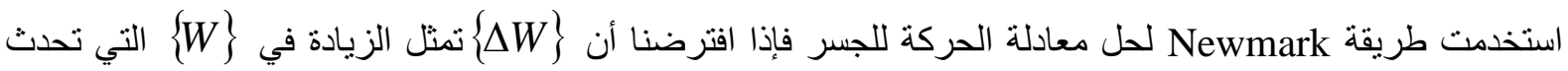
خلال فترة زمنية من $\quad$ إلى

$$
\begin{aligned}
& \{\ddot{W}\}_{t+\Delta t}=a_{0}\{\Delta W\}-a_{2}\{\dot{W}\}_{t}-a_{3}\{\ddot{W}\}_{t} \\
& \{\dot{W}\}_{t+\Delta t}=\{\dot{W}\}_{t}+a_{6}\{\ddot{W}\}_{t}+a_{7}\{\ddot{W}\}_{t+\Delta t} \\
& \{W\}_{t+\Delta t}=\{W\}_{t}+\{\Delta W\}
\end{aligned}
$$$$
\text { t+ } t \text { يمكن التعبير عنه بالمعادلات التالية: }
$$

إن المعاملات المستخدمة في طريقة Newmark تعطى كما ياتي [11] :

$a_{0}=\frac{1}{\beta(\Delta t)^{2}} \quad a_{1}=\frac{\gamma}{\beta t} \quad a_{2}=\frac{1}{\beta \Delta t} \quad a_{3}=\frac{1}{2 \beta}-1 \quad a_{4}=\frac{\gamma}{\beta}-1$

$a_{5}=\frac{\Delta t}{2}\left(\frac{\gamma}{\beta}-2\right) \quad a_{6}=\Delta t(1-\gamma) \quad a_{7}=\gamma \Delta t$

بعد حساب القيمة الابتدائية لمتجه التعجيل عند الزمن

$\{\ddot{W}\}_{0}=\left[M_{B}\right]^{-1}\left(\{F(0)\}-\left[C_{B}\right]\{\dot{W}\}_{0}-\left[S_{B}\right]\{W\}_{0}\right)$

وصباغة مصفوفة الصلابة المؤثرة

$$
\begin{aligned}
& {\left[\hat{S}_{B}\right]=\left[S_{B}\right]+a_{0}\left[M_{B}\right]+a_{1}\left[C_{B}\right]} \\
& \text { 1. حساب متجه القوة المؤثرة عند الزمن t+ t كما ياتي : }
\end{aligned}
$$

$\left\{\hat{F}_{B}\right\}_{t+\Delta t}=\left\{F_{B}\right\}_{t+\Delta t}+\left[M_{B}\right]\left(a_{0}\{W\}_{t}+a_{2}\{\dot{W}\}_{t}+a_{3}\{\ddot{W}\}_{t}\right)+$

$\left[C_{B}\right]\left(a_{1}\{W\}_{t}+a_{4}\{\dot{W}\}_{t}+a_{5}\{\ddot{W}\}_{t}\right)$

2.يتم إيجاد قيم الإزاحات عند الزمن

$\left[\hat{S}_{B}\right]\{W\}_{t+\Delta t}=\left\{\hat{F}_{B}\right\}_{t+\Delta t}$

$\{W\}_{t+\Delta t}=\left[\hat{S}_{B}\right]^{-1}\left\{\hat{F}_{B}\right\}_{t+\Delta t}$

$\{\ddot{W}\}_{t+\Delta t}=a_{0}\{\Delta W\}-a_{2}\{\dot{W}\}_{t}-a_{3}\{\ddot{W}\}_{t}$

3 ـ حساب متجهات التعجيل و السر عة كما يأتي :

$\{\dot{W}\}_{t+\Delta t}=\{\dot{W}\}_{t}+a_{6}\{\ddot{W}\}_{t}+a_{7}\{\ddot{W}\}_{t+\Delta t}$ 


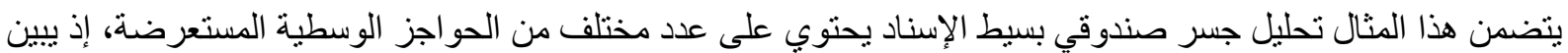

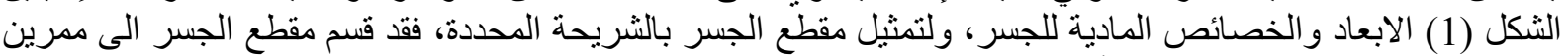

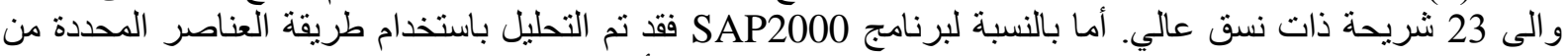

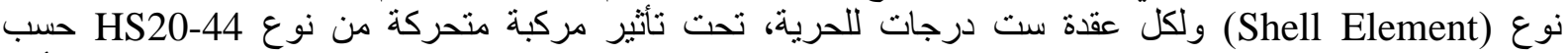

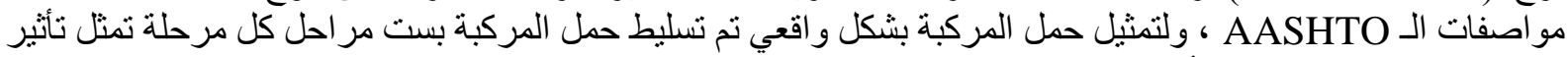

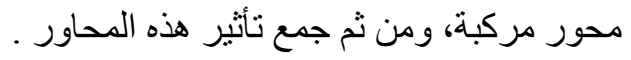

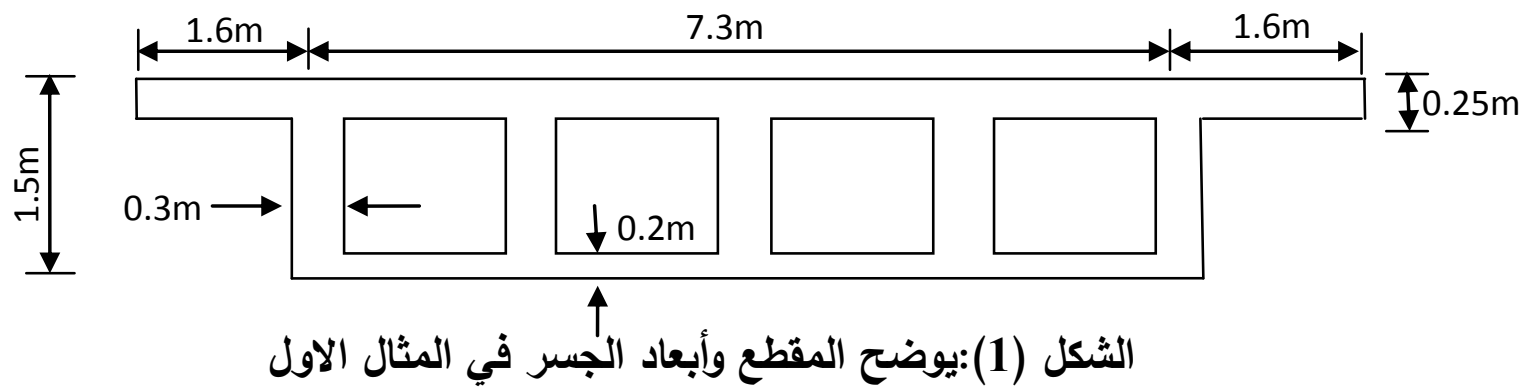

الجدول (1):يوضح الخصائص المادية للجسر

\begin{tabular}{|c|c|c|c|c|}
\hline Modulus of Elasticity & Density & $\begin{array}{c}\text { Poisson's } \\
\text { Ratio }\end{array}$ & Span & Velocity \\
\hline$E=3.34 * 10^{4} \mathrm{MPa}$ & $\rho=2400 \mathrm{~kg} / \mathrm{m}^{3}$ & $v=0.15$ & $30 \mathrm{~m}$ & $v=80 \mathrm{~km} / \mathrm{hr}$ \\
\hline
\end{tabular}

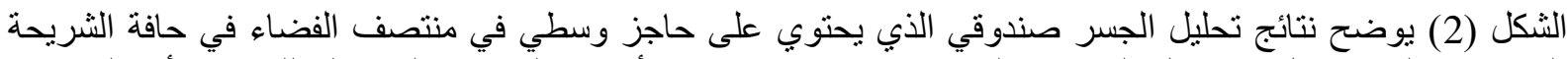

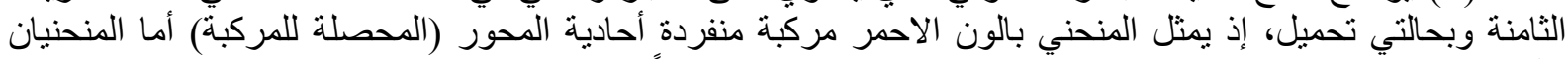

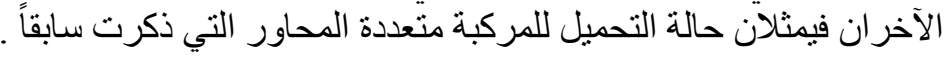

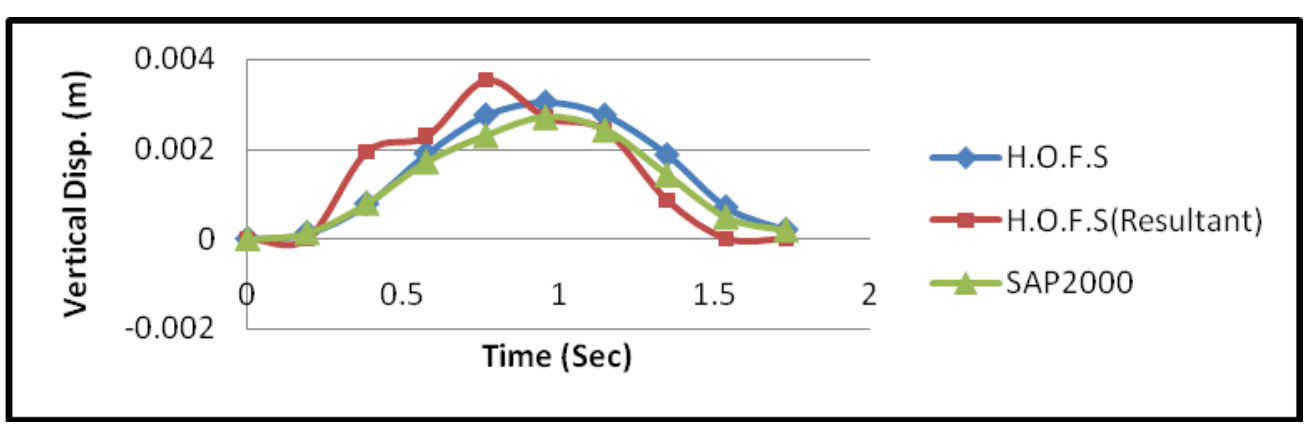

\section{الثكل (2):يوضح الاستجابة الايناميكية في منتصف فضاء}

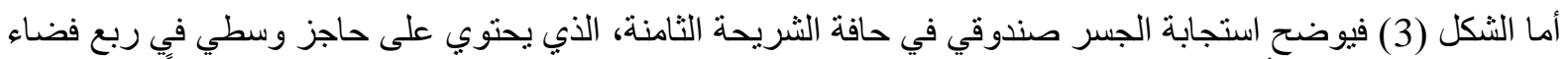

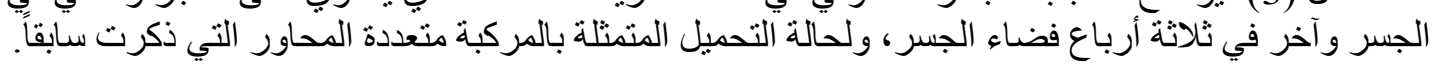




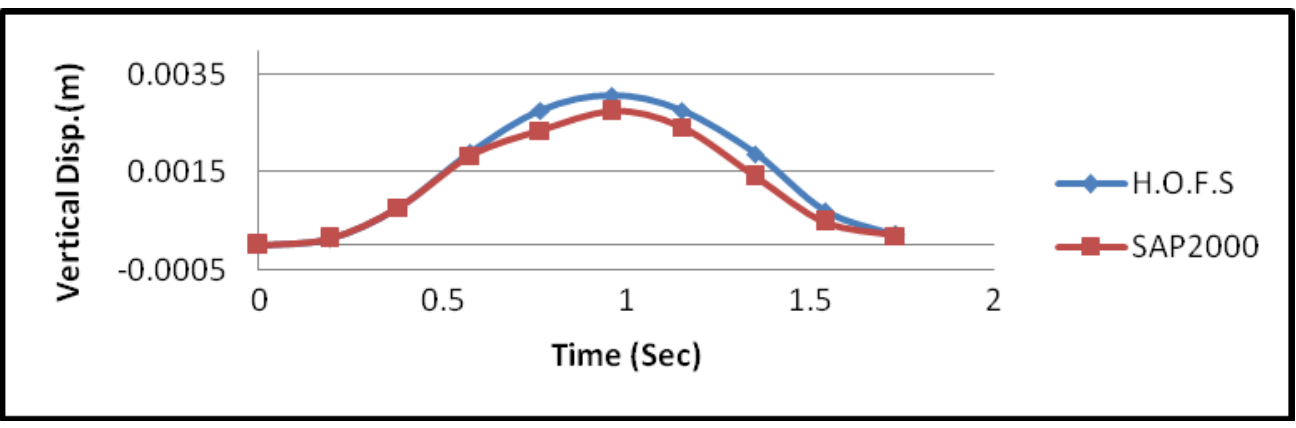

\section{الثكل (3):يوضح الاستجابة الديناميكية في منتصف فضاء الجسر}

أما الثكل (4) فيمثل الاستجابة للجسر الصندوفي في حافة الثريحة الثامنة، الذي يحتوي على ثلاثة حواجز وسطية الاول: في ربع الفضاء و الثناني في منتصف الفئ الفضاء والاخير في ثلاثة ارباع الفضاء.

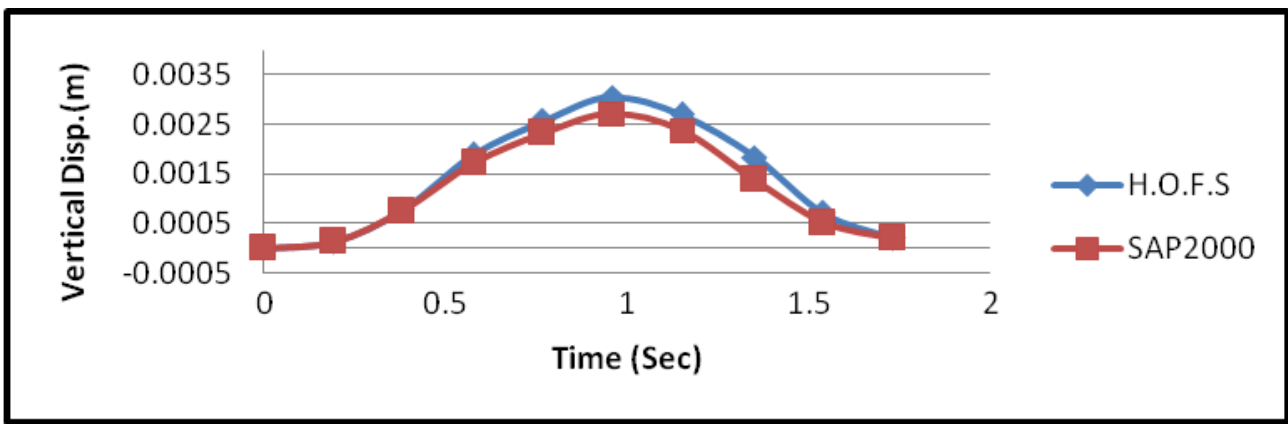

\section{الثكل (4):يوضح الاستجابة الديناميكية في منتصف فضاء الجسر}

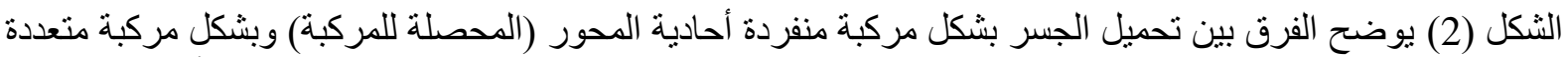

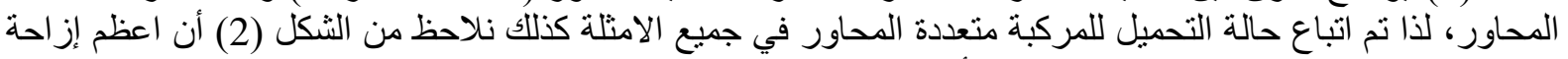

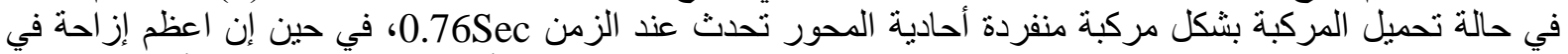

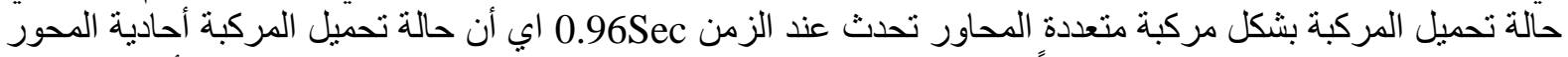

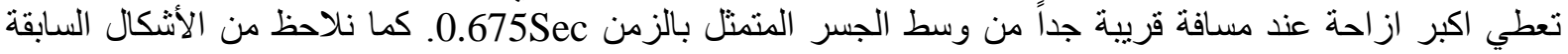
التوافق الجيد للنتائج بين طريقة الثريحة المحددة ذات النسق العالي، وطريقة العناصر المحددة (Shell Element)

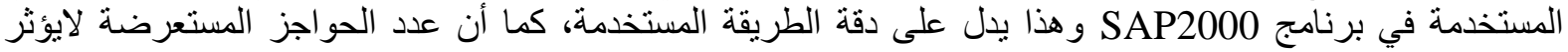
بشكل كبير وملحوظ في الاستجابة الديناميكية للجسر.

: 2.5

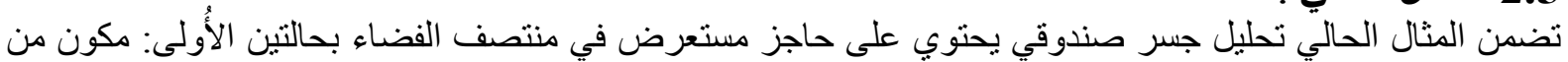

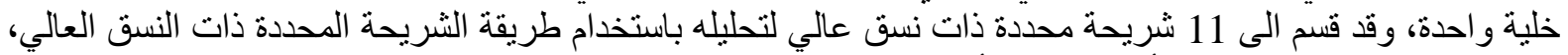

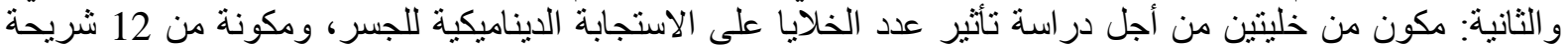

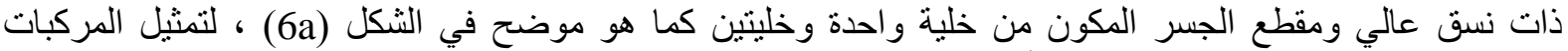

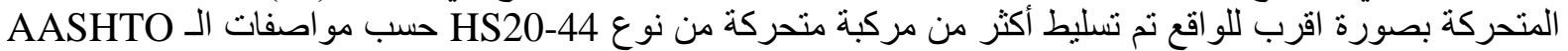

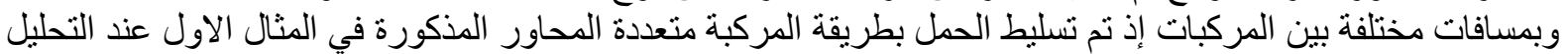
بطريقة الثريحة المحددة ذات النسق العالي. 
عبد الرزاق: التحليل الايناميكي للجسور الصندوقية بسيطة الاسناد والمحتوية على حواجز وسطية باستخدام الثريحة ـ

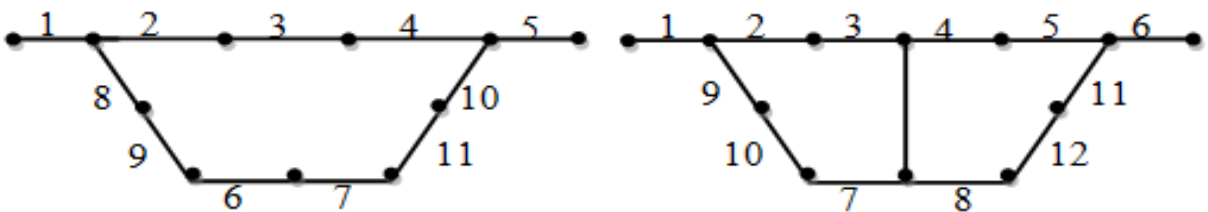

(a)

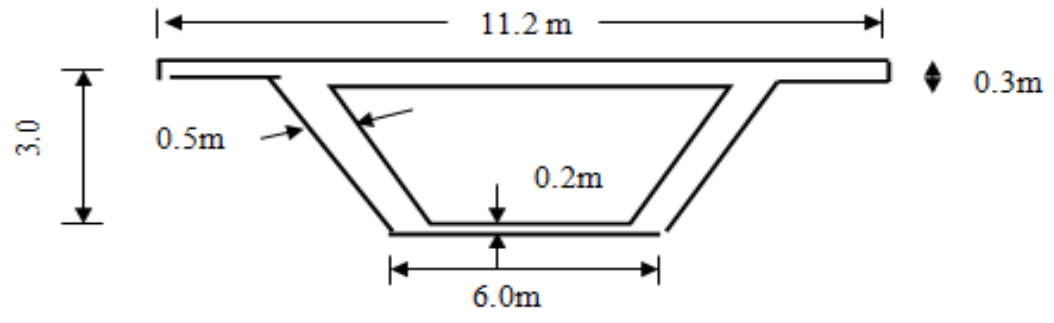

الجدول (2):يوضح الخصائص المادية للجسر

\begin{tabular}{|c|c|c|c|c|}
\hline Modulus of Elasticity & Density & $\begin{array}{c}\text { Poisson's } \\
\text { Ratio }\end{array}$ & Span & Velocity \\
\hline$E=2.1 * 10^{4} \mathrm{MPa}$ & $\rho=2400 \mathrm{~kg} / \mathrm{m}^{3}$ & $v=0.15$ & $80 \mathrm{~m}$ & $v=80 \mathrm{~km} / \mathrm{hr}$ \\
\hline
\end{tabular}

تم تحميل الجسر بالمركبتين عند تحليليه باستخدام طريقة الثريحة المحدة ذات النسق العالي وكما هو موضح في الثكل

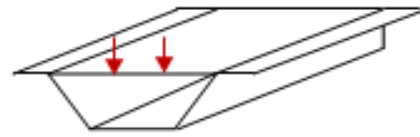

$(\mathrm{t}=0 \mathrm{sec})$

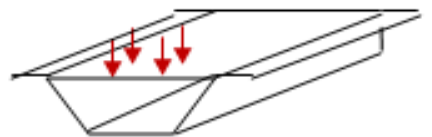

$(\mathrm{t}=0.192 \mathrm{sec})$

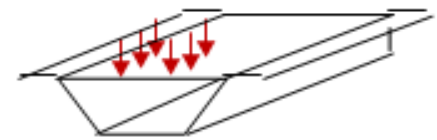

$(\mathrm{t}=0.384 \mathrm{sec})$
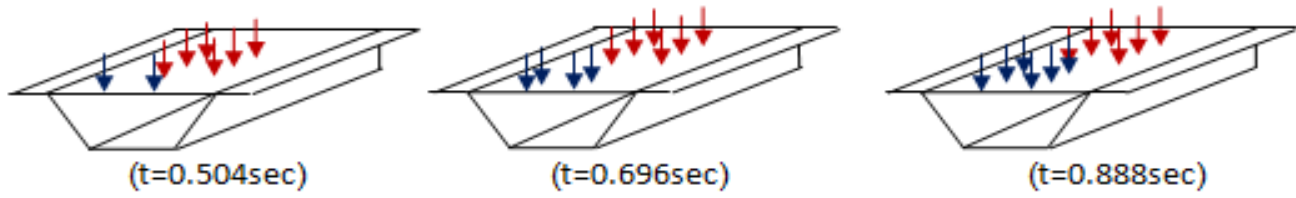

الشكل (7):يوضح كيفية تحميل الجسر بالمركبتين

الشكل (8) يوضح الاستجابة الديناميكية للجسر الصندوفي المكون من خلية واحدة وخليتين في منتصف الثفة العليا نتيجة مرور مركبة واحدة بسر عة الاسنة

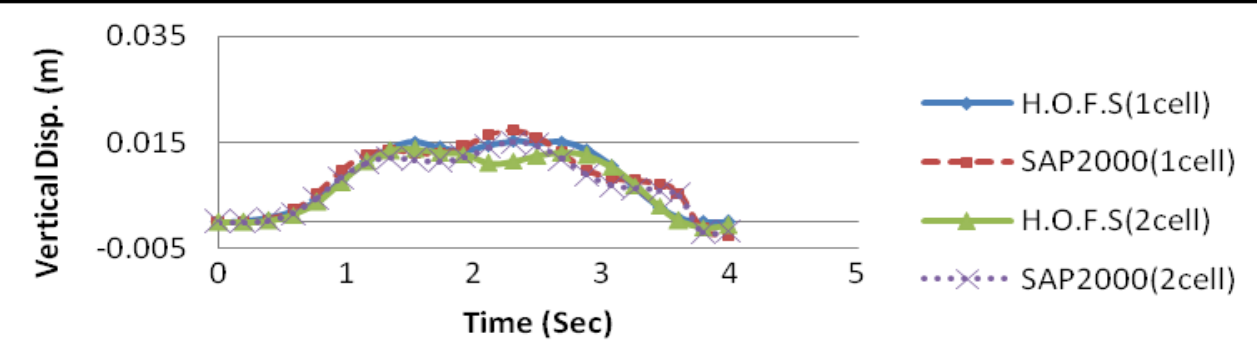

الثكل (8):يوضح الاستجابة الديناميكية في منتصف فضاء الجسر 
الثكل (9) يوضح الاستجابة الديناميكية للجسر الصندوقي المكون من خلية واحدة وخليتين في منتصف الثفة العليا،

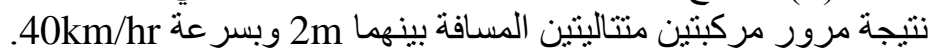

الثكل (10) يوضح الاستجابة الديناميكية للجسر الصندوقي المكون من خلية واحدة وخليتين في منتصف الثنفة العليا، نتيجة مرور مركبتين متتاليتين المسافة بينهما 4m وبسرعة 40km/hr وأن الزمن 4.56Sec يمثل الزئن الزمن الكلي لعبور

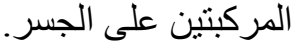

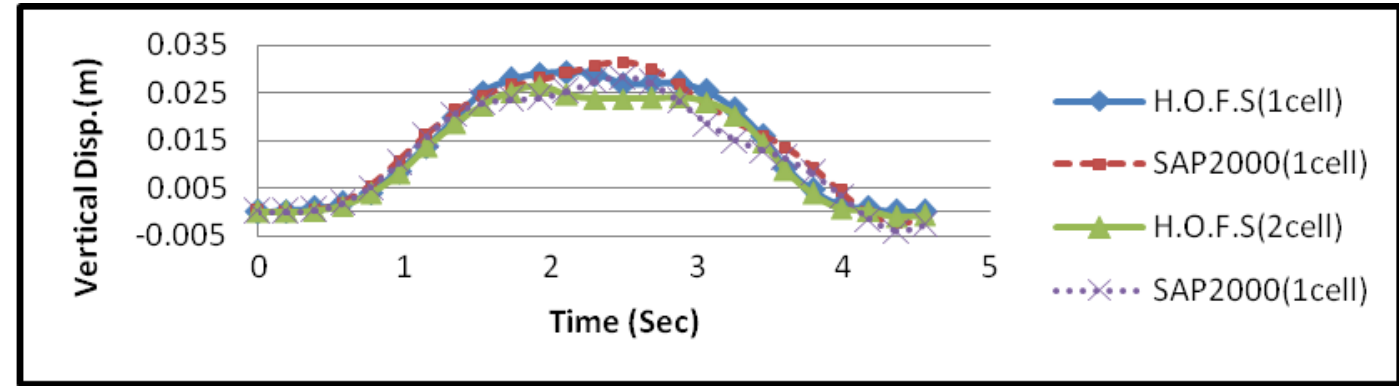

الشكل (9):يوضح الاستجابة الايناميكية في منتصف فضاء الجسر

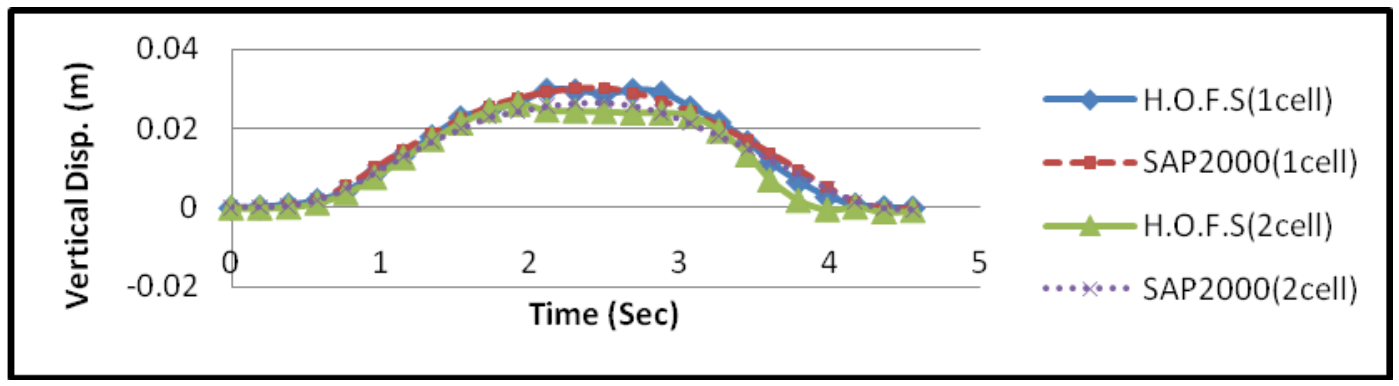

الشكل (10):يوضح الاستجابة الايناميكية في منتصف فضاء الجسر

الثكل (11) يوضح الاستجابة الديناميكية للجسر الصندوقي المكون من خلية واحدة وخليتين في منتصف الثفة العليا، نتيجة مرور مركبتين متتاليتين المسافة بينهما 6m وبسر الانيكة

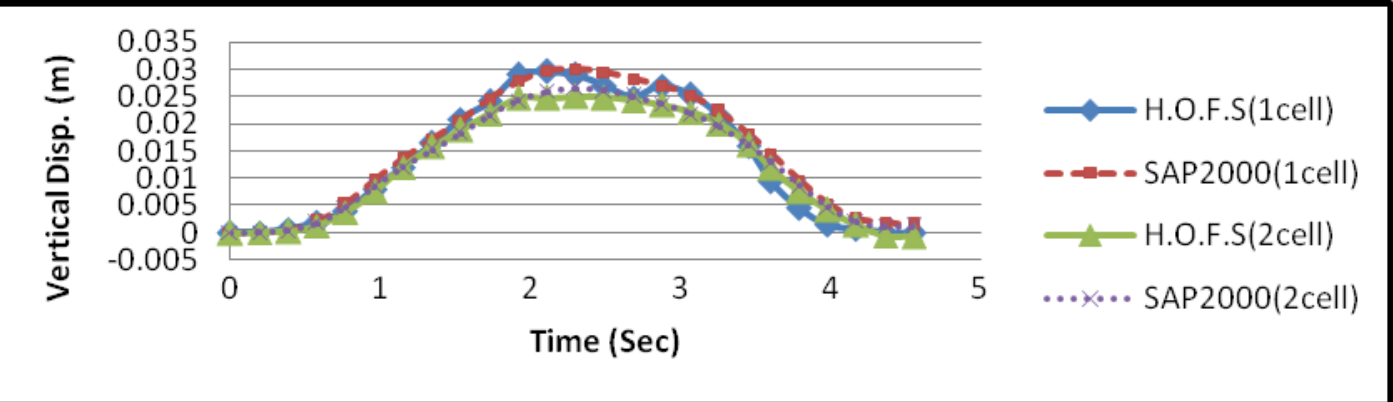

الثكل (11):يوضح الاستجابة الايناميكية في منتصف فضاء الجسر

الثكل (12) يوضح الاستجابة الديناميكية للجسر الصندوقي المكون من خلية واحدة في منتصف الثفة العليا نتيجة مرور

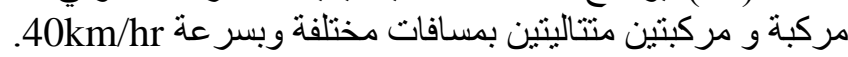


عبد الرزاق: التحليل الايناميكي للجسور الصندوقية بسيطة الاسناد والمحتوية على حواجز وسطية باستخدام الثريحة ـ

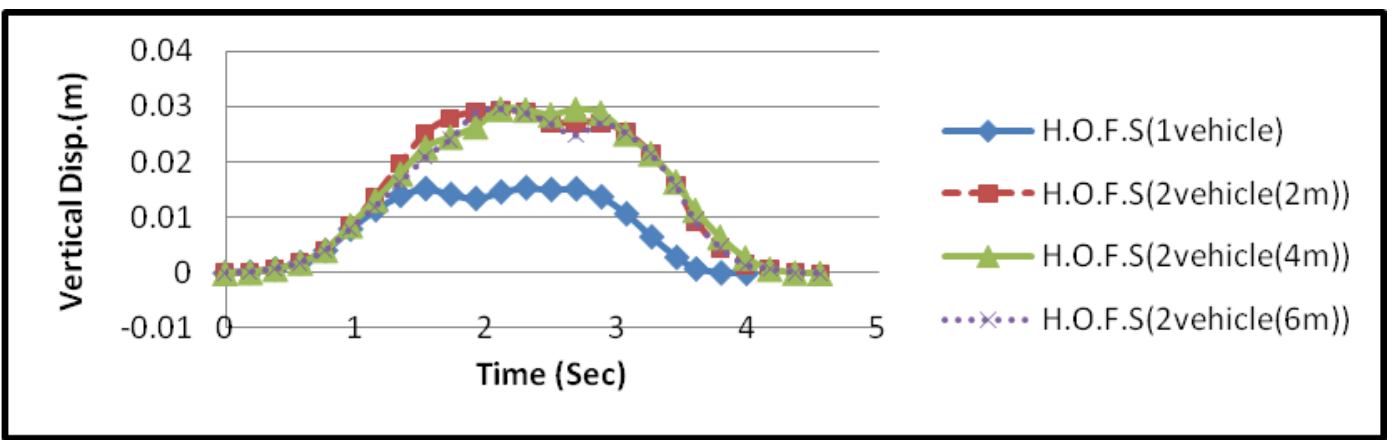

الشكل (12):يوضح الاستجابة الايناميكية في منتصف فضاء الجسر

توضح الأشكال من (8) الى (12) أن مقدار الاستجابة الديناميكية للجسر الصندوقي المكون من خليتين أقل من مقدار

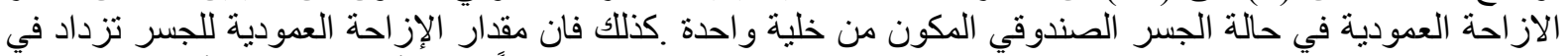

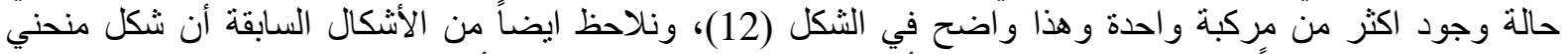

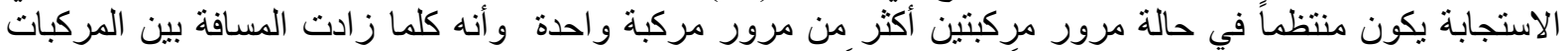

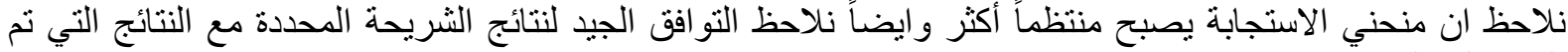

الحصول عليها من برنامج (SAP2000).

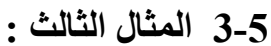

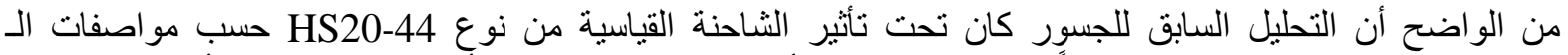
AASHTO

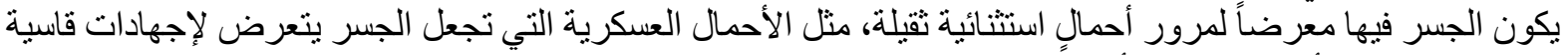

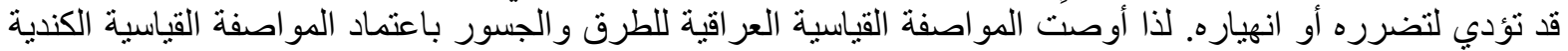

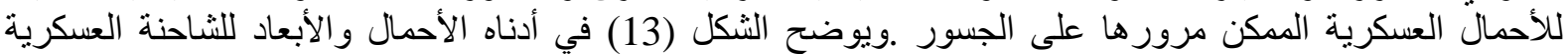
القياسية2Class 100، التي سيتم تدقيق تحمل الجسر تحت تأثير ها، فقد تم التحميل بشكل مركبة منفردة أحادية المحور

(المحصلة للمركبة).

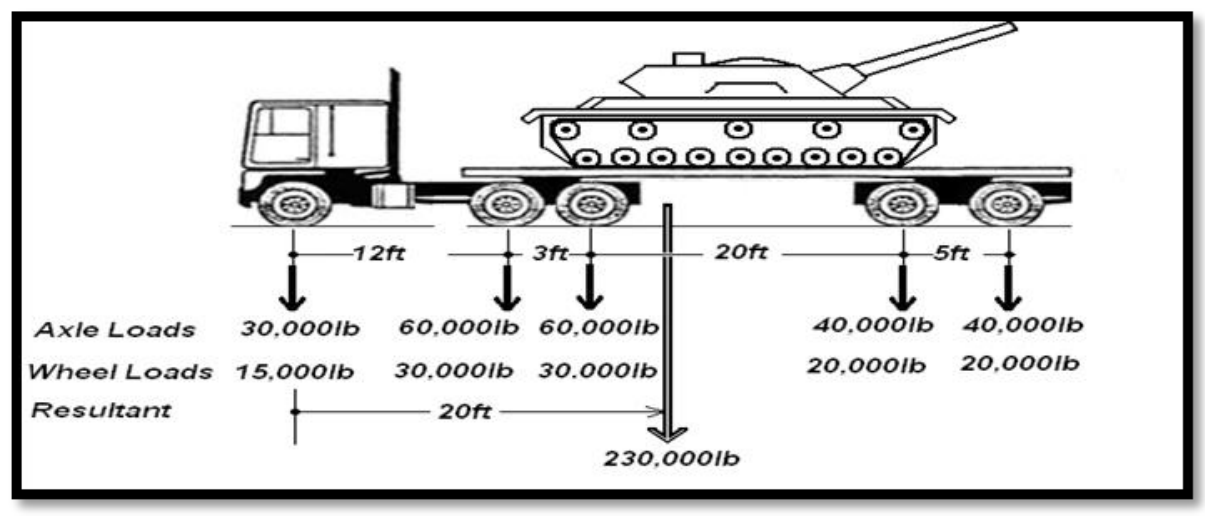

الثكل (13):يوضح نموذج المركبة المستخدم

أما عن الجسر الصندوفي فتفاصيله موضحة في الثكل (14)، فقد نم تقسيم الجسر الصندوقي المحتوي على حاجز مستعرض في وسط الفضاء الى 18 شريحة ذات نسق عالي عند تحليل الجسر باستخدام طريقة الثريحة المحددة ذات

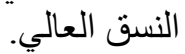




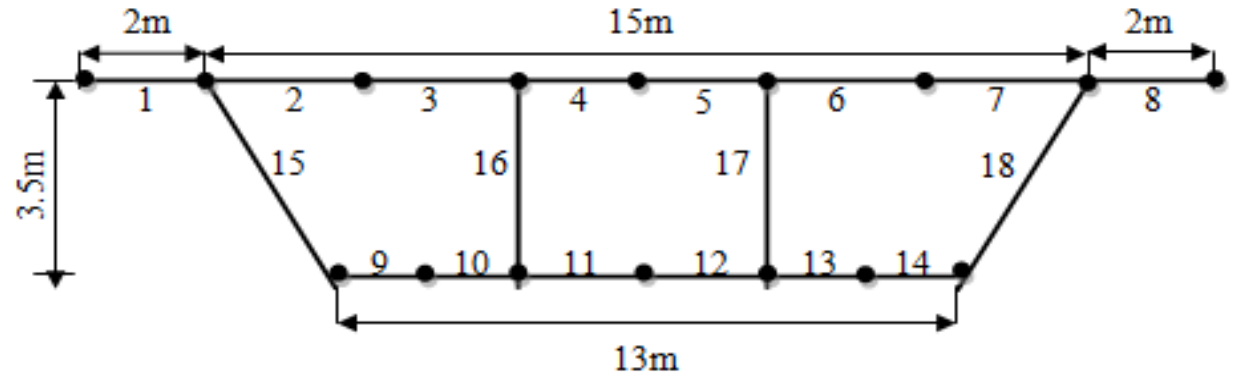

الثكل (14):يوضح مقطع الجسر وابعاده في المثال الثالث

الجدول (3):يوضح الابعاد و الخصائص المادية للجسر

\begin{tabular}{|c|c|c|c|c|}
\hline Modulus of Elasticity & Density & $\begin{array}{c}\text { Poisson's } \\
\text { Ratio }\end{array}$ & Span & Velocity \\
\hline$E=2.28^{*} 10^{4} \mathrm{MPa}$ & $\rho=2400 \mathrm{~kg} / \mathrm{m}^{3}$ & $v=0.18$ & $80 \mathrm{~m}$ & $v=100 \mathrm{~km} / \mathrm{hr}$ \\
\hline
\end{tabular}

(a)

\begin{tabular}{|c|c|c|}
\hline Top Slab Thickness & $\begin{array}{c}\text { Bottom Slab } \\
\text { Thickness }\end{array}$ & $\begin{array}{c}\text { Girder } \\
\text { Thickness }\end{array}$ \\
\hline $0.5 \mathrm{~m}$ & $0.4 \mathrm{~m}$ & $0.6 \mathrm{~m}$ \\
\hline
\end{tabular}

(b)

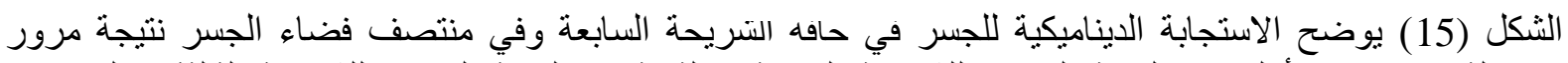

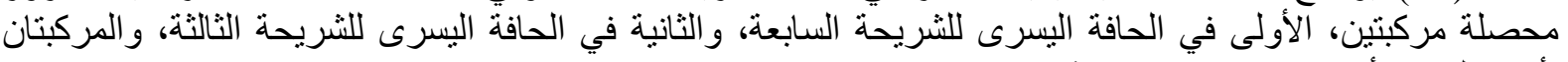

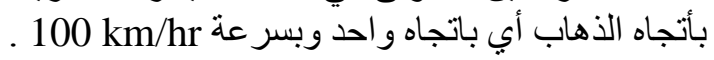

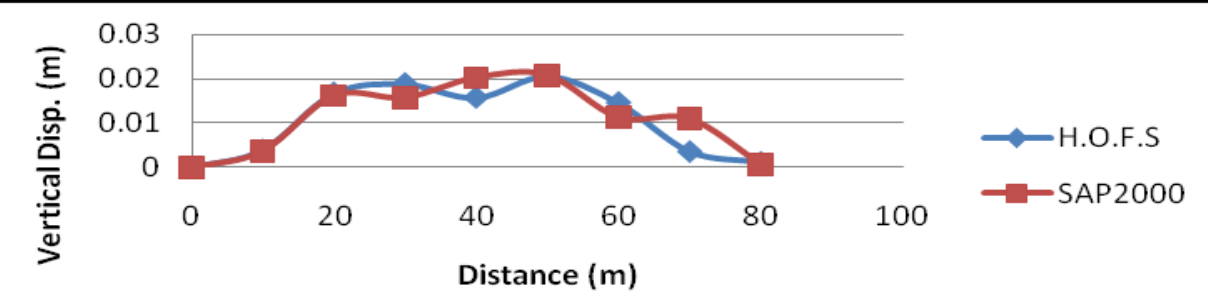

الثكل (15):يوضح الاستجابة الديناميكية في منتصف فضاء

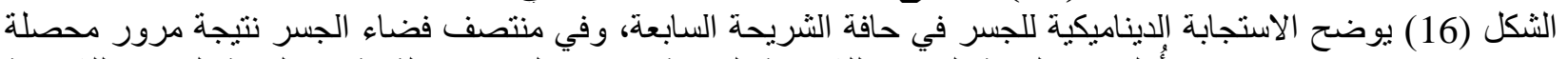

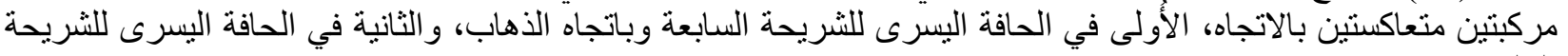

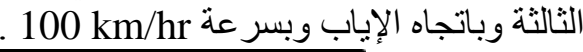

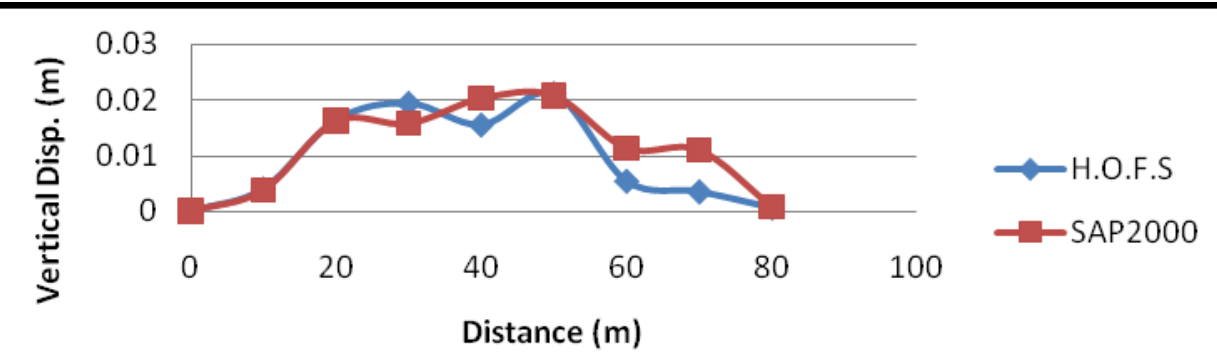

الثكل (16):بوضح الاستجابة الايناميكية في، منتصف فضاء 


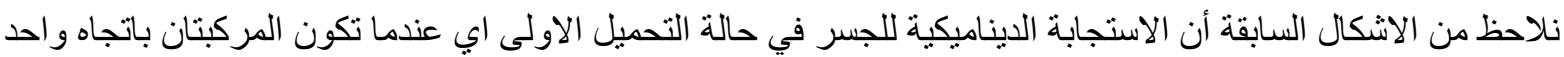

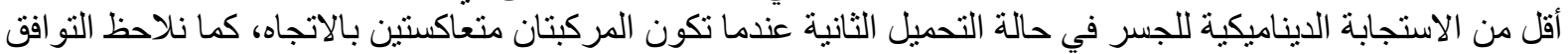

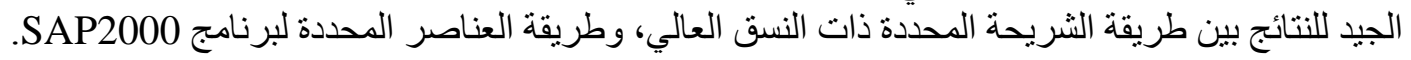

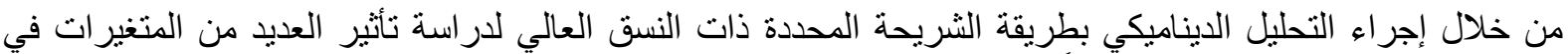

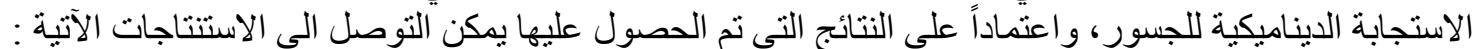

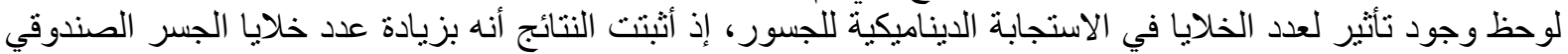

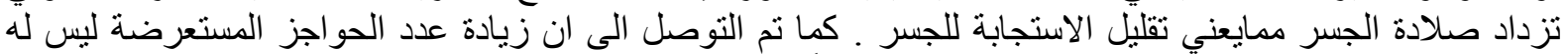

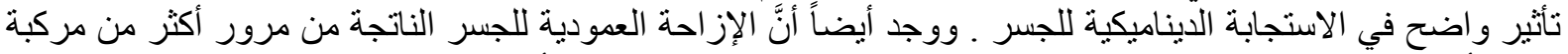

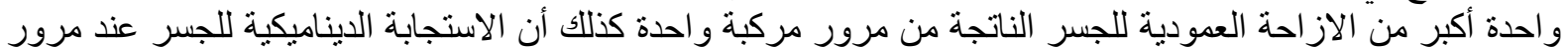

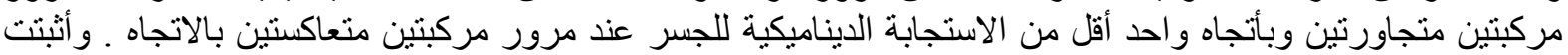

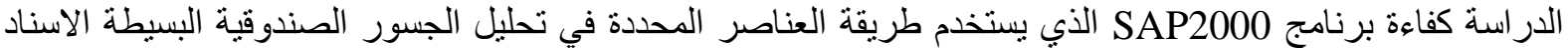
و المستمرة، إذ يتميز هذا البرنامج بالدقة و السهولة في الاستخدام و إظهار النتائج.

:References

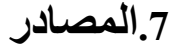

1. Abdul_Razzak, A. A. (1987). "Solution of Simply Supported and Continuous Box Girder Bridges Using Higher Order Finite Strip Method ", M.Sc. Thesis, Department of Civil Eng., University of Mosul, Iraq.

2. Abdul_Razzak, A. A. \& Mohammed, A. A. (2005). "Dynamic Analysis of Box-Girder Bridges Using A Higher Order Finite Strip Formulation", Eng. \& Technology, Vol. 24, No. 5, pp. 601-617.

3. Abdul_Razzak, A. A. \& Najem, R. M. (2002). "Solution of Curve Box-Girder Bridges With Intermediate Diaphragms", Vol 10,No.1.

4. Ali, A. H.(1999)."Dynamic Analysis of Bridges Using Finite Strip Method", Ph.D. Thesis, University of Ottawa, Ontario, Canada, pp. 1- 194.

5. AL-Shumam, B. K. A. H. (2009)."Dynamic Response of Box Girder Bridges Due to Moving Vehicles Using Finite Strip Method", M.Sc. Thesis, Department of Civil Eng., University of Mosul,Iraq.

6. Cheung, Y.K.(1969)."The Finite Strip Method in Structural Analysis", Pergamon Press, Oxford.

7. Huang, D.Z., Wang, T.L. \& Shahawy, M. (1995). "Vibration of thin- walled boxgirder bridges excited by vehicles", ASCE, J. Structural Engineering. 121(9).

8. Humar, J. L. \& Kashif, A. M. (1993). "Dynamic Response of Bridges Under Travelling Loads", Canadian J. Civil Eng. 20:287-298.

9. Loo, Y. C. \& Cusens, A. R. (1978). "The Finite Strip Method in Bridge Engineering", Eyre and Spottiswoode, London, pp.1-220.

10 Naoom, E. N. (2011)."Dynamic Analysis of Reinforced Concrete Girder Bridges Under Moving Load", Ph.D. Thesis, Department of Civil Eng., University of Mosul, Iraq.

11.Tedesco, J. W., Mcddougal W. G. \& Ross, C. A. (1999)."Structural Dynamics: Theory and Applications. Addison Wesley Longman,Inc., California.

12.Yang, Y. B. \& Wu , Y.S. (2001) . "A Versatile Element For Analyzing Vehicle Bridge Interaction Response", Engineering Structural .23:452-469.

13. Yuan-sheng, C., Cheung, Y. K. \& Au, F.T.K. (2002). "Dynamic Response of Plates Due to Moving Vehicles Using Finite Strip Method", Applied Mathematics and Mechanics, Vol. 23, No. 5, pp. 507-513.

$$
\text { تم اجراء البحث في كلية ألهندة = جامعة ألموصل }
$$

